\title{
Speed up Eulerian Video Motion Magnification
}

\author{
Yossra Hussain \\ Department of Computer Science \\ University of Technology \\ Baghdad - Iraq \\ yossra_1@yahoo.com
}

\author{
Ahmed.A.Shkara \\ Department of Computer Science \\ University of Technology \\ Baghdad - Iraq \\ ahmedsk2018@gmail.com
}

\begin{abstract}
A standard video can have small video motions that are difficult to see by naked eye because of it limited sensitivity. These hidden signals variation may have highly useful information that can be used in variety of applications fields such as healthcare, biology, mechanical engineering, civil engineering, military and security. Eulerian Video Motion Magnification is a system used to detect and amplified tiny motions in video. This system have a problem with processing time, it consumes too long time to complete the spatial_temporal analyzing.

This paper, proposes a modified approach to Speed up the processing time of Eulerian motion magnification, it minimizes the analyzing area of frames and applying the analyzing process only on a tiny motion area and ignores all unchanged background. The test results show that the proposed approach has speed up the processing time of Eulerian Motion Magnification may be to, more than $70 \%$ percentage
\end{abstract}

Keywords: Spatial-temporal analysis, Eulerian motion magnification, amplified tiny motion, motion detection, frame rate, Decomposition.

\section{INTRODUCTION}

Eulerian Video Motion Magnification is a set of simple and robust algorithms that used to extract hidden information from a video by detect and amplify spatial subtle changes [1].

Consuming too long time to complete the analyzing process is the big issue in Eulerian Video Motion Magnification and it treating as future work in other researchers [2].

The proposed approach developed to solve the processing time issue and speed up Eulerian Video Motion Magnification. It involves several techniques to achieve the above goal. The sequence of algorithm operations includes, using movement detection techniques to dedicate the first periodic frames that have a spatial subtle motion changes in a video, then image segmentation is used to drawing a mask window around tiny motion area to split it from unchanged background, and in the rest of video frames, analyzing process are applied only on the mask area, with ignoring all unchanged background.

The effectiveness of the proposed approach is demonstrated by the results of conducted tests. The processing time tests for standard data set content 4 videos (Baby breathing, Guitar, Camera and Face2) indicate that the proposed approach speed up the
Eulerian Video Motion Magnification model. For example the proposed approach show that the time processes of "Baby breathing" video speed up from 364.389340s to 116.6052s, i.e. the percentage of speed up is more than $70 \%$.

\section{Eulerian Video Motion Magnification (EVM)}

This system amplify spatial subtle changes in a video, it takes an ordinary video as input and producing it in which the tiny motions are larger to showing us hidden vital signs. The processes of system algorithms are shown in Figure (1), where combination of spatial and temporal processing is used to emphasize the subtle variations of video [3].

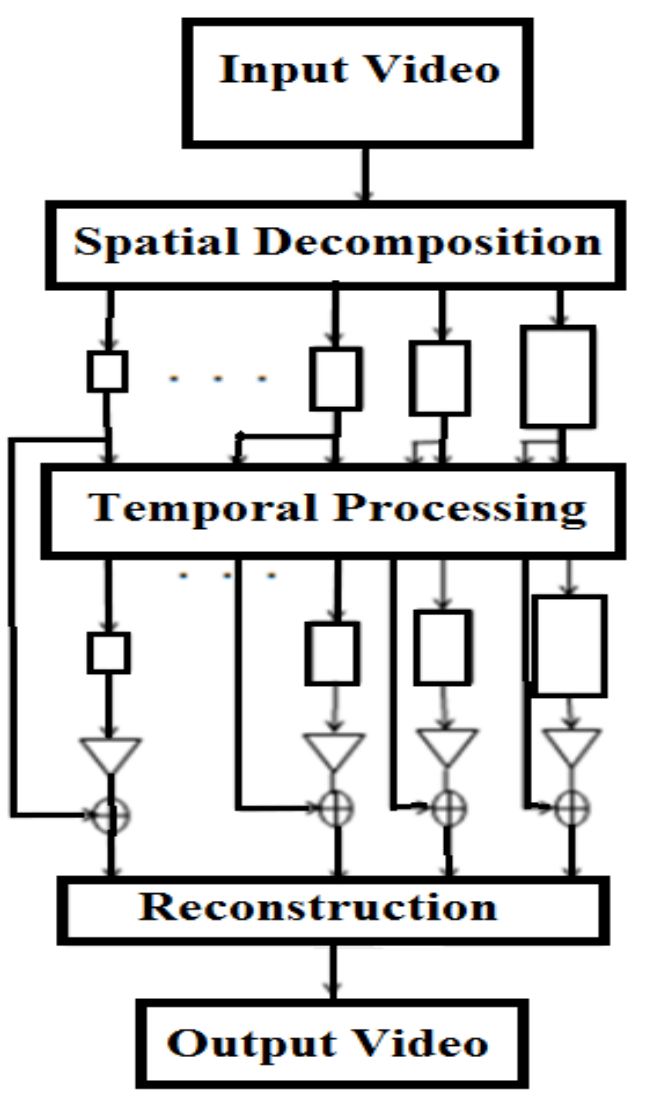

Figure 1: Eulerian Video Motion Magnification

The flowing algorithm described Eulerian Video Motion Magnification: 
Input: video

Output: amplified tiny motion in video.

\section{Step 1: Spatial Decompose:}

Start with a standard input video frames to decompose it in different spatial pool of frequencies; it apply different techniques of a video decomposition like Laplacian, Steerable and Gaussian pyramids to observe the improvement in performance of the videos.

\section{Step 2: Temporal Filtering:}

To extract hidden signals, it takes the sequence of pixel values over the time and applied temporal filtering process to the frames.

\section{Step 3: Amplified Resultant Signals:}

The resultant signals from the temporal processing are then amplified by a given factor $(\boldsymbol{\alpha})$ to reveal hidden information in the videos.

\section{Step 4: Output the Amplified Result:}

These amplified signals are added to the input signals and then a pyramid is collapsed to generate an output video.

\section{MATHEMATICAL ANALYSIS}

Read the input video frame row by row to convert it from $2 \mathrm{D}$ to $1 \mathrm{D}$, such that,

$$
\text { frame }(I)=\text { frame }(I, J)
$$

Compare between the current frame with next frame to estimate the changing in motion direction (eight directions) over the time $\delta(\mathrm{t})$.

Apply spatial displacement using a first-order Taylor series expansion by combining, frame (I), $\boldsymbol{\delta}$ (t) with amplification factor $\boldsymbol{\alpha}$ as in flow.

$$
\text { frame }(I)=\text { frame }(I) \pm(\delta(t)+\alpha)
$$

\section{RELATED WORK}

- L. Sarode , 2014 [4]: had used The (EVM) to see the movement of arm when a blood flows in it.

- M.Sushma , 2015 [5]: proposed methods of analyzing motion using time frequency analysis. In this work, address two problems: (i) Small Motion Magnification in Videos and (ii) Motion Detection in Perfusion Weighted Imaging (PWI).

- M. A. Elgharib1, 2015 [6]: Display approach-based video zoom layer that can amplify small movements within large ones. The area / layer examined is chronologically aligned and inflates the exact differences. The mat is used to enlarge the area of interest only while maintaining the safety of nearby locations. The results show larger movements, larger inflation factors and a significant reduction in the latest model.
- H.Yu Wu, 2015[7]: had used The (EVM) to see the blood flow in the face and amplify the small motions.

- $\quad$ P. Kooij, 2014[8]. An extension proposal for the binary candidate for non-gaseous filters that allows us to process pixels at very different depth layers as missing values. In his experiments he shows, the missing values must be ignored, and not inferred in the painting. It shows the results of a medical application (tremors) where it improves current baselines for maximizing movement and movement measurements.

\section{PROPOSED APPROACH}

Eulerian Video Motion Magnification system applies global motion analysis on whole frame size and the subtle motion changes happen just on a small part of a frame that construct in the same location for all video frames. The idea is, analyzing whole frames size it just time wasting. For example the video which Playback in 10 second, it consumed more than $\underline{4 \text { minute }}$ as a processing time.

The question is what is the time need to analyzing video that Playback in 2 hours? It is unpractical time. This work speed up the processing time by minimized the analyzed area. Figure (2) show the flowchart of proposed approach

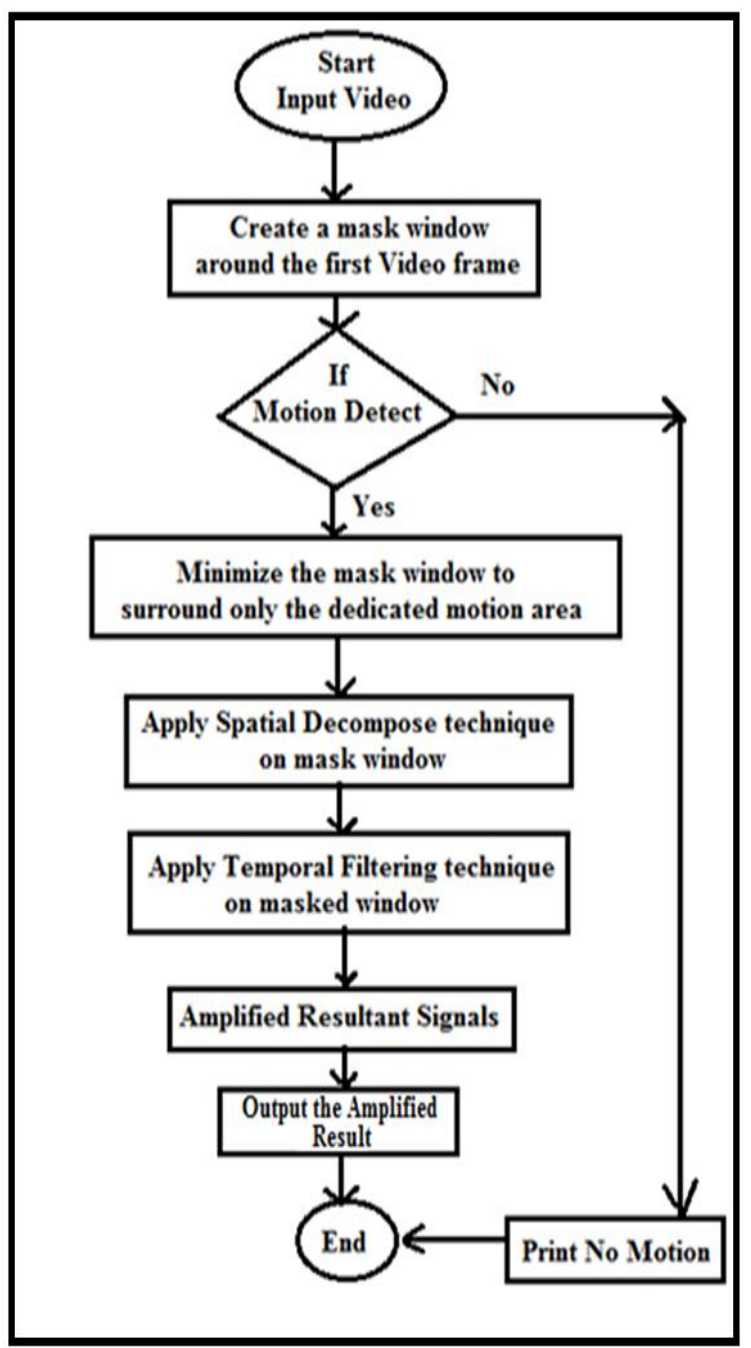

Figure 2: Flowchart of proposed approach. 
The algorithm of Speed up Eulerian Video Motion Magnification:

Input: video.

Output: amplified tiny motion in video.

Step 1: Create a mask window around the first Video frame.

//P1 (x1, y1) represent top left \& p2 (x2, y2) represent bottom down coordinate points of the mask window //

Step 2: Apply motion detection technique to detect the first tiny motion in first periodic frames.

//motion detection using background subtraction//

Step 3:Minimize the mask window to surround only the dedicated motion area.

$/ / \mathrm{p} 1(\mathrm{x} 1, \mathrm{y} 1)$, where $\mathrm{x} 1$ most top pixel \& y1 most left pixel of dedicated motion.

$\mathrm{P} 2(\mathrm{x} 2, \mathrm{y} 2)$, where $\mathrm{x} 2$ most bottom pixel \& y2 most right pixel of dedicated motion.

//

Step 4: Apply Spatial Decompose technique on mask window.

Step 5: Apply Temporal Filtering technique on masked window.

Step 6: Amplified Resultant Signals.

Step 7: Output the Amplified Result:

\section{Experimental Results}

The Frame Rate specifies how many frames are shown per second of playback of your Movies, Frame rate is most often expressed in frames per second (fps) on the device, but the rate are changes depend on the speed and power of the display device and hardware.

Video devices can display 24, 30, or 60 frames or images every second, but it typically shot at rate 24 frames per second. A minute of film shot 1,440 frames.

The proposed approach use standard data set content 4 videos for experiment the results of our method are (baby, guitar, camera and face2). All videos are tested on the proposed system using MATLAB (Version 2014b) on a (dll) laptop Intel Celeron with 2GB RAM and CPU $\mathrm{B} 710$ and $1,60 \mathrm{GHz}$ processor. Every video is taking few minutes to compute. The result of each video will be Calculate as in Baby breathing move as in flow:

Stage1: Calculate the processing time for one frame from "Baby breathing" move using (EVM).

- $\quad$ Frame Rate $=30 \mathrm{fps}$.

- No. Of Frames = 301f.

- $\quad$ Processing time for all move $=364.389340 \mathrm{~s}$.

- $\quad$ Processing time for one frame $=(435.69342 \mathrm{~s} / 301 \mathrm{f})$ $\approx 1.21054 \mathrm{~s}$.
Stage2: Calculate the processing time using a proposed approach.

- Frame size of RGB video is $(1920 * 1090) 2 \mathrm{D}=$ (2092800) 1D. Not that the all frame have the same size.

- $\quad$ The first 58 frames size is $(1920 * 1090) 2$ D, and after detected motion the next 243 frames size will be minimized by masked window to $(640 * 363) 2 \mathrm{D}$ $=(232320) 1 \mathrm{D}$

- $\quad$ The processing time for the first 58 frame $=58 \mathrm{f} *$ $1.4474 \mathrm{~s}=83.9492 \mathrm{~s}$.

- The processing time need for one new frame (minimized window size).

Size of old frame / Size of new frame (window) = time processing of old frame / time process of new frame (X). $=2092800 / 232320=1.21054 / \mathrm{X}$

$\mathrm{X} * 2092800=232320 * 1.21054 \mathrm{~s}$

$\mathrm{X}=281245.6194 / 2092800$

$\mathrm{X} \approx 0,1343 \mathrm{~s}$.

- Time processing for 243 minimized frame = number of frames $*$ time processing

$=243 * 0,1343 \approx 332.656 \mathrm{~s}$

- $\quad$ Total processing time $=$ processing time for first 55 frames + processing time for next 185 frames = $83.9492 \mathrm{~s}+332.656 \mathrm{~s} \approx 116.6052 \mathrm{~s}$

Figure (3) shows the Comparison results for all 4 videos.

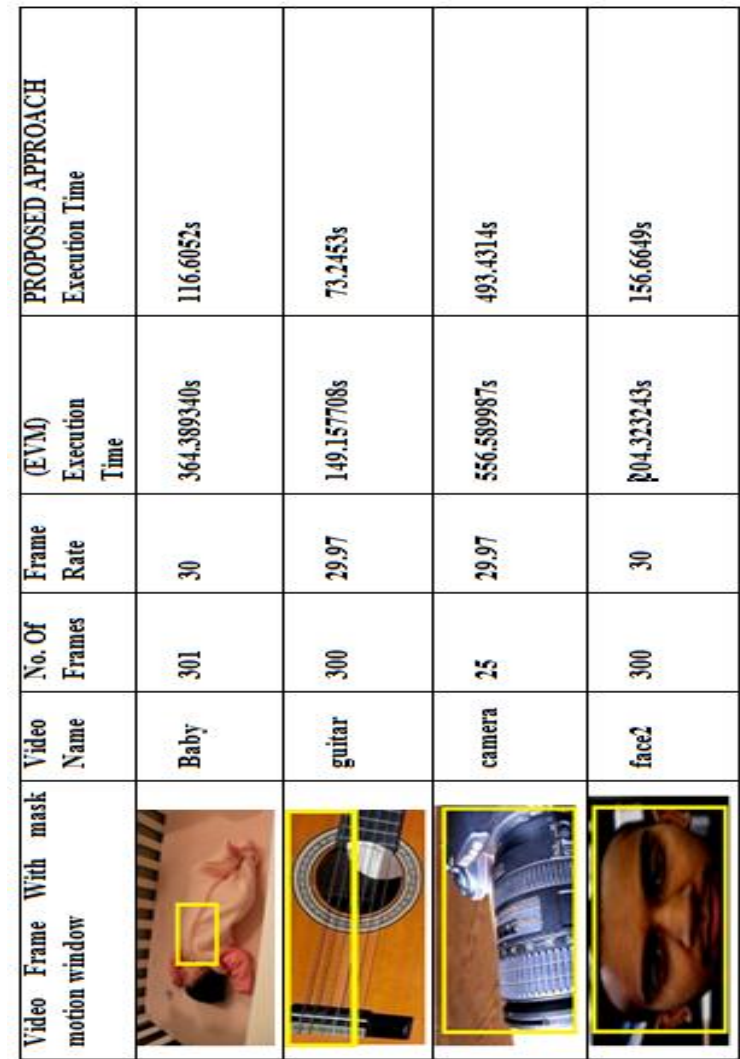

Figure 3: Comparison results on the test videos frames between traditional system and proposed approach. 


\section{CONCLUSION}

All four videos were tested on "Speed up Eulerian Video Motion Magnification" system. The proposed approach show that the processing time of "Baby" video speeds up from 364.389340s to 116.6052s, i.e. the percentage of speed up is more than $70 \%$. As shown in table 1, The percentage of speed up, is different from video to other, it depend on motion detect in each video, but the proposed approach still more practical to use in long video than the traditional one.

\section{REFERENCE}

[1] K. Kamble, N. Jagtap, A. Bhurane, "A Review: Eulerian Video Motion Magnification ", International Innovative Research in Computer and Communication Engineering (IJIRCCE), Vol. 3, Issue 3, PP. 2-6, March, 2015.

[2] C. Liu, A. Torralba, T. Freeman, H. Adelson," Motion Magnification", Quanta Research Cambridge, MIT CSAIL, PP. 1-8, 2015.

[3] R. Ayyaz, H. Javaid,"Video Colour Variation Detection and Motion Magnification to Observe Subtle Changes", M.Sc. Thesis, Electrical Engineering, Blekinge Institute of Technology, October, 2013

[4] L. Sarode, N. Mandaogade, "Video Motion Magnification using Spatio-Temporal Algorithm", International Journal of Computer Applications(IJCA), Vol. 96, PP. 9-13, June,2014.

[5] M. Sushma ,'Time Frequency Analysis for Motion Magnification and Detection" M.Sc. Thesis, Electronics and Communication Engineering ,International Institute of Information Technology, Hyderabad, INDIA, May, 2015.

[6] M. Elgharib, M. Hefeeda, T. Freeman, "Video Magnification in Presence of Large Motions", Computer Vision Foundation (CVF), IEEE Xplore, 2015.

[7] H. Yu, M. Rubinstein, S. Eugene, T. Freeman, "Eulerian Video Magnification for Revealing Subtle Changes in the World", Quanta Research Cambridge, MIT CSAIL, PP. 1-8, 2014.

[8] P. Kooij, v. Gemert, "Depth-aware Motion Magnification”, European Conference on Computer Vision (ECCV), Springer, PP. 467-482, 2016. 